\title{
Adherencia e impacto de la dieta sin gluten en niños con enfermedad celíaca
}

\author{
FRANCISCA BRAVO M. ${ }^{1}$, MARÍA PAZ MUÑOZ F. ${ }^{2-3}$ \\ 1. Becaria de Pediatría, Hospital Luis Calvo Mackenna, Universidad de Chile. \\ 2. Unidad Gastroenterología y Nutrición infantil Hospital Luis Calvo Mackenna. \\ 3. Departamento de Pediatría y Cirugía Infantil, Medicina Oriente, Universidad de Chile.
}

\begin{abstract}
Adherence and impact of gluten free diet in children with celiac disease

The treatment of Celiac Disease (CD) is a strict and permanent gluten-free diet (GFD). Factors exist that influence adherence: motivation, information, costs, correct labeling and food availability. Objectives: Describe adherence and impact of GFD in children with CD. Patients and Methods: Prospective descriptive study, through application of a 28 question survey to children 12-18 y.o. with the disorder, and parents of children 3-18 with the disease. Results: Median age: 10.5 years, 1.8 years at diagnosis. $62.5 \%$ women. Adherence to treatment was seen in $42,5 \%$ of patients according to parents, $15 \%$ according to children > 12 yrs. vs $35 \%$ parents of children $>12$ y.o. $80 \%$ of children state feeling no different on a diet, $70 \%$ find the diet hard to follow. 55\% have difficulty following the diet, among them, $42,8 \%$ are not sure what they can eat, $51.5 \%$ state it creates financial distress in the family. About $55 \%$ believe it affects family life due to the following: $55 \%$ food limitation, $50 \%$ food preparation, $75 \%$ affect eating out, 35\% affect traveling. $50 \%$ of parents have fed children a forbidden food, mainly at home (44\%). Information regarding GFD is obtained at the hospital (85\%). There is $32.5 \%$ adherence to CD foundations. Conclusions: GFD impacts children's lifestyle with difficulty in adherence and management.
\end{abstract}

(Key words: Celiac disease, gluten free diet, diet adherence).

Rev Chil Pediatr 2011; 82 (3): 191-197

\section{RESUMEN}

El tratamiento de la enfermedad celíaca (EC) es la dieta libre de gluten (DLG) estricta y permanentemente. Existen factores que influyen en adherencia: motivación, información, costos, rotulación y disponibilidad de alimentos. Objetivos: Describir adherencia e impacto de DLG en niños celíacos. Pacientes y Método: Estudio descriptivo y prospectivo con aplicación de encuesta de 28 preguntas a niños celíacos > 12 años (12-18 años) y a padres de pacientes celíacos entre 3 y 18 años. Resultados: Edad media 10,5 años y al diagnóstico

Trabajo recibido el 22 de enero de 2010, devuelto para corregir el 15 de junio de 2010, segunda versión el 11 de diciembre de 2010, tercera versión el 28 de febrero de 2011, aceptado para publicación el 23 de marzo de 2011.

Correspondencia a:

Dra. Francisca Bravo M.

E-mail: drafbravo@gmail.com 
1,8 años, 62,5\% mujeres. Adherencia 42,5\% según total de padres; $15 \%$ según niños > 12 años vs 35\% padres de niños > 12 años. El 80\% refiere no sentirse distinto, 70\% encuentra DLG difícil de seguir. 55\% le molesta seguirla porque no saben que comer (42,8\%), siendo la principal dificultad de los padres económica (51,5\%). Un 55\% cree que influye en la vida familiar: limitación alimentos (55\%), preparación especial (50\%), comer fuera de casa (75\%), viajes (35\%). El 50\% de padres ha dado alguna vez alimentos prohibidos a sus hijos, principalmente en casa (47\%). La información de DLG la obtienen en hospital (85\%). Existe 32,5\% adherencia a fundaciones celíacas. Conclusiones: La DLG implica impacto en la vida de niños celíacos, existiendo dificultades en el manejo y regular adherencia a ella.

(Palabras clave: Enfermedad celíaca, dieta libre de gluten, adherencia a la dieta).

Rev Chil Pediatr 2011; 82 (3): 191-197

\section{Introducción}

La enfermedad celíaca corresponde a una condición inmunológica que afecta principalmente al sistema gastrointestinal y que se produce secundariamente a la sensibilidad irreversible a las proteínas del gluten contenido en la dieta (Trigo-Centeno-Cebada) de pacientes predispuestos genéticamente y que cursa con atrofia intestinal severa ${ }^{1-3}$.

Se considera una enfermedad inmunológica en la que concurren factores genéticos y ambientales, habiéndose encontrado una fuerte asociación entre los genes que codifican para moléculas HLA de clase II. La enfermedad se relaciona con la presencia de heterodimero HLA DQ2 en el 95\% y DQ8 en 5-10\% ${ }^{2,4}$.

Estudios en la década pasada en Estados Unidos y Europa revelan que es una enfermedad crónica frecuente ${ }^{2}$. Se ha descrito una prevalencia en estos países de 3 a 13 por 1000 niños entre 2,5 y 15 años. En Chile la frecuencia es 1 en $3000^{1,5,6}$.

La forma de presentación varia desde síntomas mininos a severos, dependiendo de la edad, la duración y extensión de la enferme$\mathrm{dad}^{3}$. Se puede dividir en las siguientes formas clínicas: típica o clásica, atípica y asintomática o silente ${ }^{3,6-9}$.

La forma de presentación típica corresponde a un síndrome de malabsorción (diarrea crónica, baja de peso y distensión abdominal) que predomina en niños menores de 2 años. En los niños mayores la diarrea y síntomas gastrointestinales son menos frecuentes ${ }^{10}$, y se manifiestan por talla baja, retraso puberal, anemia y alteraciones de la personalidad. Estos últimos son usualmente llamados atípicos y pueden estar asociados a otras condiciones como: diabetes, anemia, osteoporosis y otras enfermedades óseas, fatiga crónica, enfermedades autoinmunes, cáncer digestivo, dermatitis herpetiforme, cambios de conducta, intestino irritable y síntomas neurológicos ${ }^{1,3,11}$. La forma asintomática o silente sólo presenta cambios histológicos en individuos aparentemente asintomáticos ${ }^{3}$.

El diagnóstico de enfermedad celíaca se realiza con la sospecha clínica, serología e histología compatible. El protocolo revisado de la Sociedad Europea de Gastroenterología Hepatología y Nutrición en 1990 confirma el diagnóstico si los síntomas son sugerentes (típico y atípicos), el screening serológico es sugerente y existe una biopsia compatible seguida de respuesta favorable al tratamiento ${ }^{1,3,12,13}$.

Los marcadores serológicos utilizados más frecuentemente son los Anticuerpos Antigliadina cuya sensibilidad y especificidad es de 90\% y 80\% respectivamente, y Antiendomisio, siendo este último más empleado debido a una especificidad y sensibilidad superior (cercana al $100 \%$ para ambos valores $)^{4}$. Sin embargo, en la actualidad se han empleando los Anticuerpos anti Transglutaminasa, con mayor sensibilidad y especificidad que los anteriores y son más efectivos en términos de disponibilidad y acceso ${ }^{1,14}$.

La lesión histológica característica es linfocitosis intraepitelial, hiperplasia de criptas y atrofia vellositaria, lo cual se traduce en atrofia intestinal que varia entre parcial a subtotal o total $^{1,3,5}$.

La detección de enfermedad celíaca tiene importantes consecuencias en la prevención 
de complicaciones asociadas a ésta y además, permite iniciar en forma precoz un tratamiento efectivo.

El tratamiento de la enfermedad celíaca consiste en una dieta libre de gluten (DLG) de por vida ${ }^{15,16}$. Esta es una dieta estricta y compleja. Estricta, ya que pequeñas cantidades ingeridas de gluten provocan atrofia vellositaria intestinal, existiendo riesgo a futuro de desarrollo de complicaciones como osteoporosis y linfoma gastrointestinal ${ }^{12,16}$. Compleja, debido a que dichas proteínas se encuentran en un sin número de alimentos básicos en la dieta chilena (Trigo-Centeno-Cebada) y en aditivos presentes tanto en alimentos elaborados como en medicamentos, existiendo además la posibilidad de contaminación cruzada al procesarse el alimento. Este es el caso de la avena que actualmente se considera libre de gluten sin embargo, se produce contaminación cruzada en cultivos conjuntos con trigo, en el proceso de cosecha, molienda y almacenaje $\mathrm{e}^{15,17}$.

En algunos países, existe la rotulación de los alimentos con terminologías como sin TACC o Gluten Free, para facilitar el tratamiento en los pacientes celíacos. En Chile, no existe obligación respecto al etiquetado de los alimentos, sólo esfuerzos de particulares y fundaciones que han iniciado la certificación nacional.

La principal limitante para el tratamiento corresponde a la adherencia, ya sea por falta de motivación por parte del paciente, falta de información o situación económica ${ }^{11}$. Otros autores señalan que las condiciones asociadas a baja adherencia son niños con diagnóstico a mayor edad, baja educación de los padres, bajo nivel socioeconómico, bajo conocimiento de la enfermedad y bajo entendimiento de sus consecuencias y complicaciones ${ }^{18,19}$.

Considerando que la dieta estricta puede afectar su bienestar psicosocial, un seguimiento cercano por personal entrenado, idealmente multidisciplinario ${ }^{20,21}$, es una buena manera de mejorar la adherencia a la terapia ${ }^{3}$.

En la literatura se describen porcentajes de adherencia a la dieta de $80 \%-95 \%$ en niños y $36-52 \%$ en adolescentes y adultos ${ }^{1,5,18,22-25}$, con un mejor porcentaje en pacientes asociados a grupos de enfermos celíacos ${ }^{26}$.
Objetivo: Describir la adherencia a la dieta sin gluten e impacto en la vida diaria de niños con EC confirmada y en control regular en un Centro Asistencial.

\section{Pacientes y Método}

Estudio descriptivo y prospectivo con aplicación de encuesta de 28 preguntas a niños celíacos > 12 años (12-18 años) y a padres de pacientes celíacos entre 3 y 18 años. El cuestionario se elaboró y fue validado previamente en la Unidad de Gastroenterología HLCM.

Se definió enfermo celíaco confirmado según criterios ESPGHAN 1990, consistentes en serología positiva (Antigliadina, Antitransglutaminasa, Antiendomisio) y biopsia compatible, con mejoría posterior al inicio de la dieta libre de gluten. Se consideró en general como alimentos permitidos al arroz, maíz y maicena, y como alimentos prohibidos al trigo, avena, cebada, centeno y gluten.

Se consideró una muestra de oportunidad que incluyó a aquellos pacientes celíacos confirmados que se mantenían en control durante el período diciembre 2007-abril 2008 en la Unidad de Gastroenterología HLCM.

Los cuestionarios fueron realizados en el domicilio de los pacientes entre Diciembre 2007 y Abril 2008 por un médico con formación en Pediatría. Se entrevistó a niños celíacos > 12 años (12-18 años) y a padres de pacientes celíacos entre 3 y 18 años por separado. Se excluyeron enfermos con co-morbilidad como Diabetes mellitus, Síndrome Down, Síndrome Turner, Síndrome Williams y retardo mental, solicitándose consentimiento y asentimiento informado según correspondiese en todos los casos.

El estudio contó con la aprobación del Comité de Ética del Hospital Luis Calvo Mackenna.

Se realizó análisis descriptivo de frecuencia y porcentajes de los datos obtenidos.

\section{Resultados}

Se controlaron 53 niños con diagnóstico confirmado de enfermedad celíaca. Se excluyeron 10 pacientes por no lograr contacto se- 
Tabla 1. Adherencia a la dieta sin gluten según padres $(n=20)$ y niños $(n=20)$ celíacos mayores de 12 años

\begin{tabular}{|lccccccccccc|}
\hline & \multicolumn{2}{c}{ Siempre } & \multicolumn{2}{c}{ Muchas veces } & \multicolumn{2}{c}{ A veces } & \multicolumn{2}{c}{ Casi nunca } & \multicolumn{2}{c|}{ Nunca } & Total \\
& $\mathbf{n}$ & $\%$ & $\mathbf{n}$ & $\%$ & $\mathbf{n}$ & $\%$ & $\mathbf{n}$ & $\%$ & $\mathbf{n}$ & $\%$ & $\mathbf{n}$ \\
Padres & 7 & 35 & 9 & 45 & 2 & 10 & 0 & 0 & 2 & 10 & 20 \\
Niños & 3 & 15 & 11 & 55 & 3 & 15 & 0 & 0 & 3 & 15 & 20 \\
\hline
\end{tabular}

gún registro telefónico y dirección y 3 por comorbilidad asociada, no hubo rechazos. Se entrevistaron 20 niños celíacos entre 12-18 años, 20 padres de niños celíacos entre 12-18 años y 20 padres de niños celíacos $<12$ años.

La edad media de los pacientes fue de 10,5 años (rango 3-18 años), edad media del diagnóstico de 1,8 años (rango 8 meses a 17 años). El $62,5 \%$ de los pacientes fue de sexo femenino.

En relación a la adherencia a la dieta sin gluten, $42,5 \%$ de todos los padres refiere que sus hijos siguen la dieta indicada siempre y un $47,5 \%$ muchas veces. Al analizar sólo a los padres de niños celíacos mayores de 12 años y compararlos con sus hijos; los padres refieren un 35\% de adherencia de sus hijos versus un $15 \%$ descrito por los niños (tabla 1).

Un 55\% de los niños y 70\% de todos los padres refiere no agradarles seguir una dieta especial, pero a un 30\% de los niños lo les importa. Ochenta por ciento de los pacientes celíacos no se siente distinto al resto de los niños por su enfermedad que es similar a la impresión de todos los padres (77,6\%).

El 70\% de todos los padres y un $82,5 \%$ de los niños consideran la dieta difícil o más menos difícil de seguir, los factores que implican dificultad para su seguimiento se encuentran descritos en la tabla 2.

El 70\% de todos los padres cree que sus hijos no han perdido eventos escolares o con amigos, en cambio el 65\% de los niños sienten haberlos perdido alguna vez. Con respecto a las amistades el $60 \%$ de los niños no cree que la dieta genere problemas en sus relaciones de amistad.

Sesenta por ciento de todos los padres y $55 \%$ de los niños refieren haber tenido problemas familiares por obligación de limitar ciertos alimentos, sintiendo que afecta la convivencia familiar en un 50 y 55\% respectivamente.

\begin{tabular}{|c|c|c|c|c|}
\hline & \multicolumn{2}{|c|}{ Padres } & \multicolumn{2}{|c|}{ Niños } \\
\hline & $n$ & $\%$ & $\mathbf{n}$ & $\%$ \\
\hline Económico & 17 & 51,5 & 2 & 14,3 \\
\hline $\begin{array}{l}\text { Ganas de comer ali- } \\
\text { mentos prohibidos }\end{array}$ & 8 & 24,3 & 4 & 28,6 \\
\hline $\begin{array}{l}\text { No le gustan los ali- } \\
\text { mentos permitidos }\end{array}$ & 4 & 12,1 & 2 & 14,3 \\
\hline $\begin{array}{l}\text { No sabe bien que } \\
\text { puede comer }\end{array}$ & 4 & 12,1 & 6 & 42,8 \\
\hline
\end{tabular}

El 50\% de los niños sienten haber tenido problemas familiares por tener que preparar comida especial $v s$ un $17,5 \%$ de todos los padres. El $75 \%$ de los niños y $60 \%$ de todos los padres sienten que la DLG afecta poder salir a comer fuera del hogar, pero sólo un tercio de padres y niños sienten que afecta el poder salir de viaje.

La tabla 3 muestra el alto conocimiento por parte de padres y niños de los alimentos permitidos y prohibidos.

El 75\% de todos los padres considera difícil encontrar tiendas con alimentos sin gluten, el $97,5 \%$ encuentra estos alimentos de alto costo económico y un $77,5 \%$ refiere no tener dificultades en leer las etiquetas de los alimentos y determinar si contienen gluten.

La información de la dieta a seguir la obtienen los padres a través de su médico tratante en el hospital (85\%) y los niños en un 55\% del médico y sólo un 35\% de familiares (tabla 4).

El 32,5\% de todos los padres refiere pertenecer a una fundación para celíacos (Convivir y/o Coacel), de éstos el 77\% llegó a la fundación por recomendación del médico tratante del hospital y un 15,3\% a través de otros enfermos. 
ENFERMEDAD CELÍACA

Tabla 3. Conocimiento de padres $(n=40)$ y niños $(n=20)$ de alimentos permitidos y prohibidos

\begin{tabular}{|c|c|c|c|c|c|c|c|c|c|c|c|c|c|c|c|c|}
\hline & \multicolumn{2}{|c|}{ Maíz } & \multicolumn{2}{|c|}{ Avena } & \multicolumn{2}{|c|}{ Centeno } & \multicolumn{2}{|c|}{ Cebada } & \multicolumn{2}{|c|}{ Trigo } & \multicolumn{2}{|c|}{ Maicena } & \multicolumn{2}{|c|}{ Gluten } & \multicolumn{2}{|c|}{ Arroz } \\
\hline & $\mathbf{n}$ & $\%$ & $\mathbf{n}$ & $\%$ & $n$ & $\%$ & $\mathbf{n}$ & $\%$ & $\mathbf{n}$ & $\%$ & $\mathbf{n}$ & $\%$ & $\mathbf{n}$ & $\%$ & $\mathbf{n}$ & $\%$ \\
\hline Padres & 1 & 3 & 35 & 88 & 35 & 88 & 34 & 85 & 39 & 98 & 6 & 15 & 36 & 90 & 0 & 0 \\
\hline Niños & 1 & 5 & 14 & 70 & 13 & 65 & 15 & 75 & 17 & 85 & 1 & 5 & 17 & 85 & 0 & 0 \\
\hline
\end{tabular}

Tabla 4. Fuentes de información de $\mathbf{4 0}$ padres y 20 niños con enfermedad celíaca

\begin{tabular}{|lcccc|}
\hline & \multicolumn{2}{c}{ Padres } & \multicolumn{2}{c|}{ Niños } \\
\hline Libros & n & \% & n & \% \\
\hline Otros enfermos & 14 & 35 & 4 & 20 \\
\hline Nutricionista & 5 & 12,5 & 0 & 0 \\
\hline Familiares & 10 & 25 & 1 & 5 \\
\hline Médico hospital & 3 & 7,5 & 7 & 35 \\
\hline Médico consultorio & 1 & 2,5 & 1 & 5 \\
\hline Fundación celíacos & 14 & 35 & 4 & 20 \\
\hline Internet & 6 & 15 & 5 & 25 \\
\hline
\end{tabular}

Tabla 5. Lugares de incumplimiento de la dieta sin gluten según padres $(n=40)$ y niños $(n=20)$ con enfermedad celíaca

\begin{tabular}{|lcccc|}
\hline & \multicolumn{2}{c}{ Niños } & \multicolumn{2}{c|}{ Padres } \\
\hline Colegio & 5 & 29,4 & 7 & 30,4 \\
Cumpleaños & 5 & 29,4 & 7 & 30,4 \\
\hline Casa amigos & 6 & 35,3 & 9 & 39,1 \\
\hline Su casa & 8 & 47 & 12 & 52,1 \\
\hline Otros lugares & 9 & 52,9 & 1 & 4,3 \\
\hline
\end{tabular}

Cincuenta por ciento de todos los padres refiere haber dado conscientemente alimentos prohibidos a sus hijos y un $75 \%$ de los niños refieren haber ingerido en forma conciente alimentos no permitidos en alguna ocasión, principalmente en el hogar (47\%). Los lugares en los cuales presentan transgresiones de la dieta se encuentran en la tabla 5.

Al preguntar a todos los padres que situación mejoraría la adherencia a la dieta de los niños, un 55\% contestó necesidad de disponer de más alimentos sin gluten en el comercio y un $40 \%$ rotulación de los alimentos libres de gluten. El 65\% de los niños marcó la primera alternativa.

De los niños encuestados un 30\% tiene familiares de primer grado con enfermedad celíaca y de estos un 58,3\% no se encuentran en control médico.

\section{Discusión}

En Chile, no existen estudios que describan la adherencia de pacientes celíacos pediátricos a DLG ni el impacto de esta en su vida diaria.

Este estudio demuestra que los porcentajes de adherencia a la dieta encontrados son bastante menores a los descritos en la literatura $^{1,5,18,22-25}$ y a su vez, que su cumplimiento se encuentra sobreestimada por lo padres. Existe una diferencia de adhesión según grupo etáreo, siendo los adolescentes más reticentes al tratamiento como lo describe la literatura. Ljungman et al, demuestran esta diferencia según edad, con un cumplimiento de $93 \%$ entre los 12-14 años cayendo a $76 \%$ entre los $15-17$ años ${ }^{5,24}$.

Se reporta que las mujeres son más cumplidoras que los varones, los niños diagnosticados menores de 4 años cumplen DLG en $80 \%$ comparados con $36 \%$ de aquellos cuyo diagnóstico fue mayor de los cuatro años ${ }^{16-19,24}$. En cuanto a la sintomatología se señala que los pacientes con formas atípicas o en aquellos cuyo diagnóstico se realizó por screening serológico presentan más transgresiones que aquellos diagnosticados con formas típicas que también se relaciona con la edad de diagnóstico ${ }^{16-27}$.

En nuestro estudio la mayoría de los pacientes sigue la dieta la mayor parte del tiempo, con algunas transgresiones ocasionales, las cuales se realizan principalmente en el hogar y en un porcentaje importante, con consentimiento de los padres, lo que traduce un desconocimiento de la enfermedad y sus consecuencias. 
La literatura demuestra como factores protectores de adherencia a la terapia la atención mediante grupos multidisciplinarlos, con supervisión y seguimiento de la dieta por nutricionista entrenada y el pertenecer a grupos de enfermos celíacos ${ }^{20-21}$. Ambas situaciones no se dan en este grupo de pacientes con un porcentaje cercano al 30\% de asesoría por nutricionista y de asociación a una fundación celíaca, pese a ser derivados luego de la primera consulta post diagnóstico.

Con respecto al impacto de la dieta en la vida diaria de los niños, la mitad de ellos refieren molestia por tener que seguir una dieta especial, pero a su vez, un casi un tercio de estos paciente refiere no importarle. La mayoría no se siente distinto al resto de los niños, pero sí consideran su dieta difícil de seguir, principalmente porque no tienen claro que pueden comer en circunstancias fuera de casa. Con respecto a los padres la mayoría considera la dieta difícil de seguir, pero la principal limitante lo constituye la situación económica, ambas limitantes descritas tanto por niños como por padres, se encuentran previamente descritas ${ }^{19,20,22}$.

Por otro lado, los niños señalan alguna vez sentirse excluidos de actividades escolares, situación desapercibida por la mayoría de los padres, aunque creen en su mayoría que la dieta no afecta sus relaciones de amistad. Con respecto a la situación familiar existen dificultades en relación a: problemas por limitación alimentos, preparación de comida especial salir a comer fuera de casa y salir de viaje ${ }^{19}$.

Un alto porcentaje de los enfermos celíacos y sus padres tienen claro los alimentos prohibidos, siendo el manejo de los padres mejor al de los niños. Una de las principales limitantes de la dieta es la dificultad en encontrar alimentos sin gluten y su alto costo, lo cual se correlaciona con la literatura ${ }^{5,20}$.

Para mejorar su calidad de vida las acciones más importantes a realizar descritas serían; el ampliar la cantidad de alimentos disponibles en el mercado y el rotular los alimentos como libres de gluten, de forma permanente ${ }^{6,18}$. Un gran porcentaje de padres refirió no tener problemas en leer una etiqueta y determinar si el producto poseía o no gluten, pero no se debe olvidar que existen alimentos que contienen sustancias prohibidas en sus aditivos o la existencia de contaminación cruzada, lo cual hace que estos alimentos puedan ser desapercibidos.

En Chile, no existe una legislación de rotulación de alimentos y no existen organismos gubernamentales encargados de medir el contenido de gluten de los alimentos. Sólo existen 2 agrupaciones de enfermos celíacos sin fines de lucro cuyas principales funciones entre otras es mantener un listado de productos nacionales y extranjeros certificados sin gluten. Es necesario se implemente un plan nacional gubernamental que apoye a los pacientes $\mathrm{y}$ fundaciones de celíacos.

La principal fuente de información con respecto a la dieta la constituye el médico tratante en el hospital. Considerando lo limitado del tiempo por control y que la educación e información con respecto a la enfermedad y la dieta son factores esenciales en asegurar una adherencia adecuada. Creemos que es imperativo disponer de nutricionistas especializadas para el asesoramiento y seguimiento de estos pacientes, y realizar talleres informativos por parte de los profesionales de salud en conjunto con las fundaciones de pacientes celíacos a sus pacientes, entorno familiar y social.

La principal limitante de este estudio es que corresponde a la realidad sólo de un porcentaje de los pacientes en control de una Unidad de Gastroenterología y por otro lado, dada la baja edad de los pacientes celíacos estudiados, sólo se pudo obtener la visión de un pequeño número de niños (20), basándose la mayor parte de la encuesta en la visión de los padres.

A su vez, nos parece importante recalcar que un $30 \%$ de los niños tenían familiares celíacos, pero más del $50 \%$ de ellos no se encontraban en control médico, demostrando así la mala adherencia tanto de los niños como de sus familiares, situación que dificulta el poder mantener una dieta adecuada a futuro y de por vida.

En conclusión, existe un porcentaje de adherencia a la dieta sin gluten más baja a la descrita en la literatura. Existe un impacto de la dieta del punto de vista emocional, social y familiar de los niños. La mayoría de los pacientes y padres tienen claro los alimentos pro- 
hibidos, pero existen ciertas limitantes como disponibilidad de alimentos y costo que hacen la dieta difícil de seguir.

Lograr mantener adherencia al tratamiento es esencial pero no fácil de lograr. Resulta fundamental mantener seguimiento continuo y multidisciplinario con monitorización por especialista, como también es deseable educación continua y pertenecer a asociaciones de pacientes celíacos que ayuden a los pacientes al entendimiento de su enfermedad.

Por último se requiere instauración de planes nacionales para apoyo a estos pacientes principalmente en la certificación de los alimentos libres de gluten y en la subvención de estos.

\section{Referencias}

1.- Hill ID, Dirks MH: Guideline for the diagnosis and treatment of celiac disease in children: recommendations of the North American Society for Pediatric Gastroenterology, Hepatology and Nutrition. J Pediatr Gastroenterol Nutr 2005; 40 (1): 1-19.

2.- Fasano A: Celiac Disease-How to Handle a Clinical Chameleon. N Engl J Med 2003; 348 (25): 68-70.

3.- Fasano C, Catassi: Celiac Disease in Children. Best Pract res Clin Gastroenterol 2005; 19 (3): 467-78.

4.- Polanco I: Celiac Disease. JPGN 2008; 47 S1: S3-S6.

5.- Guevara PG: Enfermedad celíaca. Rev Chil Pediatr 2002; 73 (4): 394-7.

6.- Araya M: Mejorar el manejo de la enfermedad celíaca. Un desafío Urgente. Rev Med Chile 2006; 134: 361-36.

7.- Cilleruelo Pascual ML, Román Riechmann E, Jiménez Jiménez J: Enfermedad celíaca silente: Explorando el iceberg en población escolar. An Esp Pediatr 2002; 57 (4): 321-6.

8.- Casellas F: Celiac disease. Med Clin (Barc) 2006; 13742.

9.- Farrell R, Kelly C: Diagnosis of celiac sprue. Am J Gastroenterol 2001; 96: 3237-46.

10.- Ludvigsson J, et al: Symptoms and Signs Have Changed in Swedish Children with coeliac disease. J Ped Gastroenterol 2004; 38: 181-6.

11.- Murray JA: The Widening Spectrum of Celiac Disease. Am J Clin Nutr 1999; 69: 354-65.

12.- Case S: The Gluten Free-Diet: How to Provide Effective Education and Resources. Gastroenterology 2005; 128: S128-34.

13.- European Society of Paediatric Gastroenterology and
Nutrition: Revised criteria for diagnosis of coeliac disease. Report of Working Group of European Society of Paediatric Gastroenterology and Nutrition. Arch Dis Child 1990; 65: 909-11.

14.- Canales, et al: Estado actual del diagnóstico y presentaciones clínicas de la enfermedad celíaca. Estudio multicéntrico. Rev Med Chile 2008; 136: 296-303.

15.- Corrao G: Mortality in patients with Coeliac Disease and their Relatives: a Cohort Study. Lancet 2001; 358 (9279): 356-61.

16.- Högberg L, Grodzinsky E, Stenhammar L: Better dietary compliance in patients with coeliac disease diagnosed in early childhood. Scand J Gastroenterol 2003; 38: 751-4.

17.- Ciclitira P, et al: Gluten-Free Diet, what is toxic? Best Practice and Research Clinical Gastroenterology 2005; 19 (3): 359-71.

18.- Celiac Disease Working Group: Federation of International Societies of pediatric Gastroenterology, hepatology, and Nutrition Consensus Report on celiac Disease. JPGN 2008; 47: 214-9.

19.- Anson $O$, et al: Celiac disease: parental knowledge and attitudes of dietary compliance. Pediatrics 1990; 85 (1): 96-103.

20.- American Gastroenterological Association Medical Position Statement: Celiac sprue. Gastroenterology 2001; 120: 1522-5.

21.- Rashid M, et al: Celiac Disease: Evaluation of the Diagnosis and Dietary Compliance in Canadian Children. Pediatrics 2005; 116: e754-9.

22.- Mayer M, Greco L, Troncone R: Compliance of adolescents with celiac disease with a gluten-free diet. Gut 1991; 32: 881-5.

23.- Fabiani E, Catassi C, Villari A, Gismondi P, Pierdomenico $R$, Rätsch IM: Dietary compliance in screeningdetected coeliac disease adolescents. Acta Paediatr Suppl 1996; 412: 65-7.

24.- Ljungman $G$, Myrdal U: Compliance in teenagers with celiac disease-a Swedish follow-up study. Acta Paediatr 1993; 82: 235-8.

25.- Pietzak M: Follow-up of Patients With Celiac Disease: Achieving Compliance With Treatment. Gastroenterol 2005; 128: S135-41.

26.- Marsh M: Gluten major histocompatibility and the small intestine. A molecular and immunologic approach to the spectrum of gluten sensibility. Gastroenterology 1992; 102: 330-54.

27.- Fabiani E, Taccari LM, Rätsch I-M, Di Giuseppe S, Coppa GV, Catassi C: Compliance with gluten-free diet in adolescents with screening-detected celiac disease: a 5-year follow-up study. J Pediatr 2000; 136: 841-3. 